\title{
A new construction of the Ree groups of type ${ }^{2} G_{2}$
}

\author{
Robert A. Wilson \\ School of Mathematical Sciences, \\ Queen Mary, University of London, \\ Mile End Road, London E1 4NS, U.K.
}

Submitted 25/02/08; revised 30/03/09

\begin{abstract}
We give a new elementary construction of Ree's family of finite simple groups of type ${ }^{2} G_{2}$, which avoids the need for the machinery of Lie algebras and algebraic groups. We prove that the groups we construct are simple of order $q^{3}\left(q^{3}+1\right)(q-1)$ and act doubly transitively on an explicit set of $q^{3}+1$ points, where $q=3^{2 k+1}$. Moreover, our construction is practical in the sense that generators for the groups and many of their maximal subgroups may be easily obtained.
\end{abstract}

\section{Introduction}

The two families of Ree groups, namely $R\left(3^{2 k+1}\right)={ }^{2} G_{2}\left(3^{2 k+1}\right)$ and $R\left(2^{2 k+1}\right)=$ ${ }^{2} F_{4}\left(2^{2 k+1}\right)$, are among the least understood of the finite simple groups. Partly this is due to the difficulty of constructing them. They were the last infinite families of finite simple groups to be discovered (see Ree's 1961 paper [6]), and the standard construction is described in Carter's 1972 book [3], using a great deal of technical machinery. Put simply, they are described as subgroups of $G_{2}(q)$ and $F_{4}(q)$ acting on the respective Lie algebra, of dimension 14 or 52 , centralizing certain automorphisms of order 2 which are constructed with considerable technical difficulty.

It is well-known, however, that the smallest representation of $G_{2}(q)$ (for $q$ odd), and also of $R\left(3^{2 k+1}\right)$, has dimension 7 , and can be obtained from the octonion algebra. Similarly, the smallest representation of $F_{4}(q)$ (for $q$ prime to 3 ), and also of $R\left(2^{2 k+1}\right)$, has dimension 26 , and can be obtained from an exceptional quadratic Jordan algebra. Explicit constructions of these representations are given for example by Howlett, Rylands and Taylor [5] (see also [7]), and are included in MAGMA [2]. Nevertheless, these constructions require even more machinery, namely some representation theory of Lie algebras and/or algebraic groups.

There is also a geometrical construction of ${ }^{2} G_{2}(q)$ (but not of ${ }^{2} F_{4}(q)$ ) due to Tits (see [8], and also [9]), which works with 6-dimensional projective space and describes explicitly a set of $q^{3}+1$ points permuted 2-transitively by the group. However, his list of points, given by complicated formulae, is obtained from the Lie theory. Of course, one can take this list of points as a definition, and deduce the required properties of the group from it, but then there is no motivation for this seemingly arbitrary definition.

In this paper I shall give a completely elementary construction of the small Ree groups, ${ }^{2} G_{2}(q)$, which uses no Lie theory whatsoever. This is related to the Tits construction, but I believe offers several significant advantages over the latter. What is new here is, firstly, the observation that the entire construction is determined by elementary properties of the affine group $2^{3}: 7: 3$; secondly, a simple algebraic 
definition of the points from which Tits's formulae can be easily derived by solving equations; and, thirdly, a simple algebraic criterion for membership in the group, from which the group order and simplicity follow easily. Thus, our viewpoint is quite different, and perhaps more importantly, it provides a template for the much more difficult construction of the large Ree groups, ${ }^{2} F_{4}\left(2^{2 n+1}\right)$, which is described in a forthcoming paper [10]. The latter construction is, I believe, completely new. To demonstrate the utility of these constructions, I calculate the group orders, prove simplicity, and construct most of the maximal subgroups.

\section{A definition of the Ree groups}

The affine group $H:=2^{3}: 7: 3 \cong A \Gamma L_{1}(8)$ may be built from the field of order 8 by taking the split extension of the additive group by the multiplicative group, and then further extending by the field automorphisms. It has a natural module of degree 7 , generated by the following linear transformations with respect to a basis $\left\{i_{t} \mid t \in \mathbb{F}_{7}\right\}$ of the underlying vector space $V$ over any field $F$ of characteristic not 2:

$$
\begin{array}{ll}
\alpha & : \quad i_{t} \mapsto \pm i_{t} \\
\beta \quad: \quad & i_{t} \mapsto i_{t+1} \\
\gamma \quad: \quad & i_{t} \mapsto i_{2 t}
\end{array}
$$

It is easy to see, either from first principles or by calculating the character, that on restriction to the subgroup $2^{3}: 7$ the exterior square $\Lambda^{2}(V)$ of $V$ is a direct sum of three copies of the restriction of $V$. It follows easily that $\Lambda^{2}(V)$ itself has a unique quotient module isomorphic to $V$, and that the natural quotient map (defined up to an overall scalar factor) is $m: i_{t} \wedge i_{t+r} \mapsto i_{t+3 r}$ for $r$ a quadratic residue mod 7 (that is, $r=1,2,4)$. Similarly there is a unique submodule, $V^{\prime}$, say, isomorphic to $V$, and the inclusion map is (again up to a scalar factor) $\mu: i_{t} \mapsto \sum_{r=1,2,4}\left(i_{t+r} \wedge i_{t+3 r}\right)$.

The map $m$ may be interpreted as the (anti-commutative) multiplication $i_{t} i_{t+r}=$ $i_{t+3 r}$ for $r=1,2,4$ which defines the 8-dimensional octonion algebra with basis $\left\{1, i_{t}\right\}$ over $F$, and this leads to a quick and easy definition of $G_{2}(F)$ as the group of automorphisms of this algebra. The map $\mu$ is the corresponding comultiplication, with respect to the natural bilinear form under which $\left\{i_{t}\right\}$ is an orthonormal basis. It is a triviality to verify that the composite map $m \mu$ is multiplication by the scalar 3 . Hence there is a fundamental dichotomy between the characteristic 3 case, when $m \mu=0$, and the rest, when $m \mu$ is an $H$-module automorphism of $V$. If the characteristic of $F$ is not 3 , then $\Lambda^{2}(V)=\operatorname{im} \mu \oplus \operatorname{ker} m$; and if the characteristic of $F$ is 3 , then $\operatorname{im} \mu \subset \operatorname{ker} m$.

We assume from now on that $F$ has characteristic 3 , and define $V^{\prime}=\operatorname{im} \mu$ and $W=\operatorname{ker} m$. Now we can construct the $H$-module $V^{*}:=W / V^{\prime}$, and observe that $V^{*}$ is also isomorphic, as an $H$-module, to $V$. To see this isomorphism clearly, pick the basis $\left\{i_{t}^{\prime}, i_{t}^{*} \mid t \in \mathbb{F}_{7}\right\}$ of $W$ by

$$
\begin{aligned}
i_{t}^{\prime}=\mu\left(i_{t}\right) & =\sum_{r=1,2,4} i_{t+r} \wedge i_{t+3 r} \\
& =i_{t+1} \wedge i_{t+3}+i_{t+2} \wedge i_{t+6}+i_{t+4} \wedge i_{t+5} \\
i_{t}^{*} & =i_{t+1} \wedge i_{t+3}-i_{t+2} \wedge i_{t+6}
\end{aligned}
$$

Then it is a triviality that $\beta: i_{t}^{\prime} \mapsto i_{t+1}^{\prime}$ and $\beta: i_{t}^{*} \mapsto i_{t+1}^{*}$ and $\gamma: i_{t}^{\prime} \mapsto i_{2 t}^{\prime}$, and it is an easy exercise to show that $\gamma: i_{t}^{*} \mapsto i_{2 t}^{*}-i_{2 t}^{\prime}$ and $\alpha: i_{t}^{\prime} \mapsto \pm i_{t}^{\prime}$ and $\alpha: i_{t}^{*} \mapsto \pm i_{t}^{*}$, with a + sign just for $t=1,2,4$.

With the above notation we may define natural $H$-module homomorphisms $\theta$ : $V \rightarrow W: i_{t} \mapsto i_{t}^{\prime}$ (which is an embedding) and $\rho: W \rightarrow V: i_{t}^{*} \mapsto i_{t}, i_{t}^{\prime} \mapsto 0$ (which 
Table 1: The new basis of $W$

\begin{tabular}{c|cc|}
$r$ & $v_{r}^{\prime}$ & $v_{r}^{*}$ \\
\hline-3 & $v_{0} \wedge v_{-3}+v_{-2} \wedge v_{-1}$ & $v_{-3} \wedge v_{-2}$ \\
-2 & $v_{1} \wedge v_{-3}+v_{-2} \wedge v_{0}$ & $v_{-1} \wedge v_{-3}$ \\
-1 & $v_{-3} \wedge v_{2}+v_{-1} \wedge v_{0}$ & $v_{-2} \wedge v_{1}$ \\
0 & $v_{-3} \wedge v_{3}+v_{2} \wedge v_{-2}+v_{1} \wedge v_{-1}$ & $v_{-3} \wedge v_{3}+v_{-2} \wedge v_{2}$ \\
1 & $v_{3} \wedge v_{-2}+v_{0} \wedge v_{1}$ & $v_{2} \wedge v_{-1}$ \\
2 & $v_{-1} \wedge v_{3}+v_{0} \wedge v_{2}$ & $v_{1} \wedge v_{3}$ \\
3 & $v_{3} \wedge v_{0}+v_{2} \wedge v_{1}$ & $v_{3} \wedge v_{2}$ \\
\hline
\end{tabular}

is a projection). The former is (by definition) essentially the comultiplication $\mu$ on $V$, so we may define $G_{2}(F)$ as the subgroup of the orthogonal group on $V$ (where $\left\{i_{t}\right\}$ is an orthonormal basis) which commutes with $\theta$.

To define the Ree groups we twist $\rho$ by a field automorphism to give $\rho^{*}: \lambda^{*} i_{t}^{*} \mapsto$ $\lambda i_{t}$, where $\lambda^{*}=\lambda^{3^{k+1}}$ if $F$ has order $3^{2 k+1}$. Thus ${ }^{* *}$ denotes the field automorphism $\lambda \mapsto \lambda^{3}$. Then the Ree group $R(F)$ or ${ }^{2} G_{2}(F)$ (also known as $R\left(3^{2 k+1}\right)$ or ${ }^{2} G_{2}\left(3^{2 k+1}\right)$ ) is defined as the subgroup of $G_{2}(F)$ consisting of elements which commute with $\rho^{*}$.

\section{Generators for the Ree groups}

The basis $\left\{i_{0}, \ldots, i_{6}\right\}$ of $V$ exhibits a symmetry group $2^{3}: 7: 3$, but to see other symmetries it is helpful to change basis on $V$ to $\left\{v_{0}, v_{ \pm 1}, v_{ \pm 2}, v_{ \pm 3}\right\}$ defined as follows:

$$
\begin{array}{ccc}
v_{-3}=-i_{3}-i_{5}-i_{6} & & v_{3}=i_{3}-i_{5}+i_{6} \\
v_{-2}=-i_{1}-i_{2}-i_{4} & & v_{2}=i_{1}+i_{2}-i_{4} \\
v_{-1}=-i_{0}-i_{3}+i_{6} & & v_{1}=-i_{0}+i_{3}-i_{6}
\end{array}
$$

[This basis is almost completely determined by the requirements that it is a symplectic basis with respect to which $\alpha$ is diagonal and $\gamma$ is unitriangular.] We can calculate $\theta\left(v_{r}\right)$ explicitly and for simplicity of notation define $v_{r}^{\prime}=\theta\left(v_{r}\right)$. These vectors are listed in the second column of Table 1 . The third column gives a choice of $v_{r}^{*}$ such that $\rho\left(v_{r}^{*}\right)=v_{r}$. Notice that with respect to these new (ordered) bases of $V$ and $W$, both $\theta$ and $\rho$ are graded. The calculations required to verify the correctness of this table are relegated to the Appendix.

What symmetries are visible with respect to this new basis? First notice that the element $\alpha$ which negates $i_{0}, i_{3}, i_{5}, i_{6}$ acts by negating $v_{ \pm 1}, v_{ \pm 3}$. Then the element $\alpha^{\beta^{3}}$ which negates $i_{1}, i_{2}, i_{3}, i_{6}$ acts by negating $v_{0}$ and swapping $v_{r}$ with $v_{-r}$ for $r \neq 0$.

Next consider a diagonal element $\delta:=\operatorname{diag}\left(\lambda_{-3}, \ldots, \lambda_{3}\right)$, defined with respect to the new basis. For this to commute with $\theta$, the following equations hold:

$$
\begin{aligned}
\lambda_{-3} & =\lambda_{0} \lambda_{-3}=\lambda_{-2} \lambda_{-1} \\
\lambda_{0} & =\lambda_{-3} \lambda_{3}=\lambda_{-2} \lambda_{2}=\lambda_{-1} \lambda_{1}
\end{aligned}
$$

which imply that $\delta=\operatorname{diag}\left(\lambda_{-3}, \lambda_{-2}, \lambda_{-3} \lambda_{-2}^{-1}, 1, \lambda_{-3}^{-1} \lambda_{-2}, \lambda_{-2}^{-1}, \lambda_{-3}^{-1}\right)$. We easily check that this element $\delta$ does indeed commute with $\theta$, for any choice of non-zero $\lambda_{-3}$, $\lambda_{-2}$ in $F$.

Now we need $\delta$, or equivalently its $3^{k+1}$ th power, $\delta^{*}$, to commute also with $\rho^{*}$. We calculate the image of $v_{-3}^{*}$ under $\delta^{*} \rho^{*}$ to be $\lambda_{-3}^{*} v_{-3}$, whereas the image under 
$\rho^{*} \delta^{*}$ is $\lambda_{-3} \lambda_{-2} v_{-3}$. Therefore $\lambda_{-3}^{*}=\lambda_{-3} \lambda_{-2}$ and so $\lambda_{-2}=\lambda_{-3}^{3^{k+1}-1}$. Similarly we calculate the images of the other basis elements, and check that they all give the same equation. Therefore the diagonal elements

$$
\delta(\lambda):=\operatorname{diag}\left(\lambda, \lambda^{3^{k+1}-1}, \lambda^{-3^{k+1}+2}, 1, \lambda^{3^{k+1}-2}, \lambda^{-3^{k+1}+1}, \lambda^{-1}\right)
$$

belong to the Ree group $R(q)$, for any $\lambda \in F=\mathbb{F}_{q}$, where $q=3^{2 k+1}$.

In fact, these diagonal elements together with the group $H \cong 2^{3}: 7: 3$ generate $R(q)$ provided $q>3$. However, this is not obvious, and we shall not use it in the sequel. The cyclic group $\{\delta(\lambda) \mid \lambda \in F\}$ is of course none other than the maximally split torus. It is inverted by $\alpha^{\beta^{3}}$ so $\left\langle\delta(\lambda), \alpha^{\beta^{3}}\right\rangle \cong D_{2(q-1)}$.

\section{A definition of the Ree unital}

We say that $v \in V$ is a $*$-vector, or $v \in v^{*}$, if $v^{*} \equiv v \wedge w \quad\left(\bmod V^{\prime}\right)$ for some $w \in V$. Similarly, we say that $\langle v\rangle$ is a $*$-point (or just point, if there can be no confusion) if $v$ is a $*$-vector. The Ree unital (also known as the Ree-Tits unital) is the set of $*$-points. The reason for the notation $v \in v^{*}$ is that $v \wedge w$ corresponds (via duality) to the 2 -space $\langle v, w\rangle$.

In order to classify the $*$-vectors, we first analyse the leading terms: writing $v=\sum_{r=-3}^{3} \alpha_{r} v_{r}$ we say $\alpha_{r} v_{r}$ is the leading term (or $r$ is the grade of $v$ ) if $r$ is maximal such that $\alpha_{r} \neq 0$. Similarly, if also $w=\sum_{r=-3}^{3} \beta_{r} v_{r}$ then the leading term of $v \wedge w$ is $\alpha_{r} \beta_{s} v_{r} \wedge v_{s}$, where $r$ and $s$ are maximal such that $\alpha_{r} \neq 0$ and $\beta_{s} \neq 0$. Note in particular that this leading term is uniquely determined. More generally, define the leading term of an arbitrary vector $\sum_{r=-3}^{3} \sum_{s=-3}^{3} \gamma_{r s} v_{r} \wedge v_{s}$ in $\Lambda^{2}(V)$ to be the sum $\sum_{r+s=t} \gamma_{r s} v_{r} \wedge v_{s}$, where $t$ is maximal subject to this sum being non-zero. Now consider the leading term in $v \wedge w$ : this cannot lie in $V^{\prime}$, since no vector in $V^{\prime}$ has a single leading term. (For example, if the leading term was $v_{2}^{\prime}=v_{-1} \wedge v_{3}+v_{0} \wedge v_{2}$ there would have to be a term in either $v_{3} \wedge v_{0}$ or $v_{3} \wedge v_{2}$, which is a contradiction.) Now the grading of the maps $\theta$ and $\rho$ implies that the leading term in $v$, which corresponds with the leading term in $v^{*}$, is also the leading term in $v \wedge w$, so by inspection of Table 1 , this is either $v_{-3}$ or $v_{3}$.

So unless $v=v_{-3}$ we may assume that $v=v_{3}+\sum_{r=-3}^{2} \alpha_{r} v_{r}$ and $w=$ $\sum_{r=-3}^{2} \beta_{r} v_{r}$ and then look in turn at the coefficients of $v_{3}^{*}, \ldots, v_{-3}^{\prime}$ in the congruence $v^{*} \equiv v \wedge w \quad\left(\bmod V^{\prime}\right)$ to solve for one coefficient at a time in terms of the parameters $\alpha_{2}, \alpha_{1}, \alpha_{0}$. The equations are as follows, in the order they are used:

\begin{tabular}{lll} 
Term & Equation & Solved for \\
\hline$v_{3}^{*}$ & $\beta_{2}=1$ & $\beta_{2}$ \\
$v_{2}^{*}$ & $\beta_{1}=-\alpha_{2}^{*}$ & $\beta_{1}$ \\
$v_{3}^{\prime}$ & $\beta_{0}=\alpha_{1} \beta_{2}-\alpha_{2} \beta_{1}$ & $\beta_{0}$ \\
$v_{2}^{\prime}$ & $\beta_{-1}=\alpha_{0} \beta_{2}-\alpha_{2} \beta_{0}$ & $\beta_{-1}$ \\
$v_{1}^{*}$ & $\alpha_{1}^{*}=\alpha_{2} \beta_{-1}-\alpha_{-1} \beta_{2}$ & $\alpha_{-1}$ \\
$v_{1}^{\prime}$ & $\beta_{-2}=\alpha_{1} \beta_{0}-\alpha_{0} \beta_{1}$ & $\beta_{-2}$ \\
$v_{-1}^{*}$ & $\beta_{-1}^{*}=\alpha_{-2} \beta_{1}-\alpha_{1} \beta_{-2}$ & $\alpha_{-2}$ \\
$v_{0}^{*}$ & $\alpha_{0}^{*}=-\beta_{-3}+\alpha_{-2} \beta_{2}-\alpha_{2} \beta_{-2}$ & $\beta_{-3}$ \\
$v_{-1}^{\prime}$ & $\alpha_{2} \beta_{-3}-\alpha_{-3} \beta_{2}=\alpha_{-1} \beta_{0}-\alpha_{0} \beta_{-1}$ & $\alpha_{-3}$ \\
\hline$v_{0}^{\prime}$ & $\beta_{-3}=\alpha_{2} \beta_{-2}-\alpha_{-2} \beta_{2}+\alpha_{1} \beta_{-1}-\alpha_{-1} \beta_{1}$ \\
$v_{-2}^{\prime}$ & $\alpha_{-3} \beta_{1}-\alpha_{1} \beta_{-3}=\alpha_{-2} \beta_{0}-\alpha_{0} \beta_{-2}$ & \\
$v_{-3}^{\prime}$ & $\alpha_{-3} \beta_{0}-\alpha_{0} \beta_{-3}=\alpha_{-2} \beta_{-1}-\alpha_{-1} \beta_{-2}$ & \\
$v_{-2}^{*}$ & $\alpha_{-2}^{*}=\alpha_{-1} \beta_{-3}-\alpha_{-3} \beta_{-1}$ & \\
$v_{-3}^{*}$ & $\alpha_{-3}^{*}=\alpha_{-3} \beta_{-2}-\alpha_{-2} \beta_{-3}$ & \\
\hline
\end{tabular}


[Actually, this only works as it stands if $\beta_{1} \neq 0$, that is $\alpha_{2} \neq 0$. Otherwise, the equation labelled $v_{-1}^{*}$ cannot be solved for $\alpha_{-2}$ : in this case, take the difference of the equations labelled $v_{0}^{*}$ and $v_{0}^{\prime}$ to get an equation in which the coefficient of $\alpha_{-2}$ is $\beta_{2}=1$. The second half of the table contains the equations which are not used, except in this special case.]

We conclude that there are at most $q^{3}+1$ possibilities for $v$, up to scalar multiplication, or $(q-1)\left(q^{3}+1\right)$ altogether. We shall show in the next section that there are at least this number. At that point it would be possible to identify this copy of the Ree unital with the one given in [8], and hence deduce all the required properties of the group from that source. However, we gain some advantage by using the underlying algebraic structure we have produced rather than relying solely on the geometry.

\section{2-transitivity}

In order to prove that there are exactly $q^{3}+1$ points, and that the group acts 2-transitively on them, we explicitly construct some automorphisms described by lower unitriangular matrices, which fix the point $\left\langle v_{-3}\right\rangle$, and permute the other $q^{3}$ points regularly. Indeed, if $\phi$ is such an automorphism, we may choose the values of $\alpha_{2}, \alpha_{1}$ and $\alpha_{0}$ in $\phi\left(v_{3}\right)=v_{3}+\sum_{r=-3}^{2} \alpha_{r} v_{r}$ arbitrarily, and then the method of the previous section provides enough equations to solve for every entry in the matrix of $\phi$.

The easiest case is when $\alpha_{0}=1$ and $\alpha_{1}=\alpha_{2}=0$. We obtain the element $s_{1}$ which centralizes $v_{-3}, v_{-2}, v_{-1}$ and maps

$$
\begin{aligned}
& v_{0} \mapsto v_{0}-v_{-3} \\
& v_{1} \mapsto v_{1}+v_{-2} \\
& v_{2} \mapsto v_{2}-v_{-1}-v_{-3} \\
& v_{3} \mapsto v_{3}+v_{0}+v_{-2}+v_{-3}
\end{aligned}
$$

The case $\alpha_{1}=1$ and $\alpha_{0}=\alpha_{2}=0$ is actually the element $\beta$ of $H$, which gives an alternative method of calculating it. This element (call it $s_{2}$ ) centralizes $v_{-3}, v_{-2}$ and maps

$$
\begin{aligned}
v_{-1} & \mapsto v_{-1}-v_{-3} \\
v_{0} & \mapsto v_{0}+v_{-2} \\
v_{1} & \mapsto v_{1}+v_{-3} \\
v_{2} & \mapsto v_{2}-v_{0}+v_{-2} \\
v_{3} & \mapsto v_{3}+v_{1}-v_{-1}+v_{-3}
\end{aligned}
$$

The third case is $\alpha_{2}=1$ and $\alpha_{0}=\alpha_{1}=0$. This element $s_{3}$ centralizes $v_{-3}$ and maps

$$
\begin{aligned}
v_{-2} & \mapsto v_{-2}-v_{-3} \\
v_{-1} & \mapsto v_{-1}+v_{-2}-v_{-3} \\
v_{0} & \mapsto v_{0}-v_{-1} \\
v_{1} & \mapsto v_{1}+v_{0}+v_{-1}+v_{-3} \\
v_{2} & \mapsto v_{2}-v_{1}-v_{0}-v_{-1}+v_{-3} \\
v_{3} & \mapsto v_{3}+v_{2}-v_{-1}+v_{-2}-v_{-3}
\end{aligned}
$$

Indeed, $s_{1}=\left[s_{3}, s_{2}\right]$, so it suffices to check $s_{3}$. The calculations required to check that $s_{3}$ is in the Ree group are relegated to the Appendix. Once we have verified that these three linear transformations do indeed commute with $\theta$ and $\rho^{*}$, we have shown that indeed the three parameters $\alpha_{2}, \alpha_{1}$ and $\alpha_{0}$ can be chosen from $F$ independently, and therefore there are exactly $q^{3}+1$ points in the Ree unital. 
Moreover, the stabilizer of the point $\left\langle v_{-3}\right\rangle$ is transitive on the remaining $q^{3}$ points. Since $\alpha^{\beta^{3}}$ maps $v_{-3}$ to $v_{3}$, the Ree group is 2 -transitive on the $q^{3}+1$ points.

\section{The order of the group}

Lemma 1 The stabilizer in $R(q)$ of the point $\left\langle v_{-3}\right\rangle$ consists of lower triangular matrices with respect to the basis $\left\{v_{-3}, \ldots, v_{3}\right\}$.

Proof. If $\left\langle v_{-3}\right\rangle$ is fixed, then so is $\left\langle v_{-3}^{*}\right\rangle$ which corresponds to the 2 -space $\left\langle v_{-3}, v_{-2}\right\rangle$. Therefore also $\left\langle v_{-3}^{*}, v_{-2}^{*}\right\rangle$ is fixed, and so is the space spanned by the corresponding 2 -spaces, that is $\left\langle v_{-3}, v_{-2}, v_{-1}\right\rangle$. Together with their orthogonal complements, these form a complete composition series of the vector space $V$.

Let $B$ be the subgroup generated by the lower unitriangular matrices $s_{1}, s_{2}, s_{3}$ and the diagonal matrices $\delta(\lambda)$. Thus $B$ is a group of lower triangular matrices, and has order at least $q^{3}(q-1)$. On the other hand, the argument in Section 3 shows that the stabilizer of the two points $\left\langle v_{-3}\right\rangle$ and $\left\langle v_{3}\right\rangle$ is cyclic of order $q-1$, and therefore the order of $B$ is exactly $q^{3}(q-1)$.

Corollary 1 The group $B$ is the full stabilizer of the point $\left\langle v_{-3}\right\rangle$ in $R(q)$ and has order $q^{3}(q-1)$.

In particular we have proved:

Theorem 1 The order of the group $R(q)$ is $q^{3}\left(q^{3}+1\right)(q-1)$, where $q=3^{2 k+1}$ is the order of the underlying field.

\section{Simplicity}

With this preparation simplicity can be proved using one of the standard variants of Iwasawa's Lemma:

Lemma 2 If $G$ is a perfect group acting faithfully and primitively on a set $\Omega$, and the point stabilizer has a normal soluble subgroup whose conjugates generate $G$, then $G$ is simple.

Theorem 2 The group $R(q)$, for $q=3^{2 k+1}$, is simple if $q>3$.

Proof. In this case the point stabilizer $B$ is itself soluble, and it is a triviality that $R(q)$ is generated by conjugates of the point stabilizer, since $R(q)$ acts 2transitively and therefore primitively. To prove that $R(q)$ is perfect, provided $q>3$, observe that $(q-1) / 2$ is odd, and every odd-order element in the maximal torus is a commutator, since the normalizer of the torus is $D_{2(q-1)}$. Moreover, provided $q>3$, the commutator subgroup of $B$ is the full Sylow 3-subgroup, of order $q^{3}$. On the other hand, the Sylow 2-subgroup has order $2^{3}$, and its normalizer contains $2^{3}: 7: 3$, so the involutions are also commutators. Therefore $B$ is contained in $R(q)^{\prime}$, so $R(q)$ is perfect, so by the lemma is simple.

\section{The involution centralizer}

Now the involution $\alpha=\delta(-1)=\operatorname{diag}(-,+,-,+,-,+,-)$ centralizes $\delta(\lambda), s_{2}$ and $\alpha^{\beta^{3}}$. Together these generate a group isomorphic to $2 \times L_{2}(q)$, acting as the orthogonal group $\Omega_{3}(q)$ on the 3 -space $\left\langle v_{-2}, v_{0}, v_{2}\right\rangle$. 
On the other hand, the centralizer of the involution in the point stabilizer $B$ has order $q(q-1)$, so there are $q^{2}$ involutions in $B$, and the involution fixes exactly $q+1$ points, so the number of involutions in $R(q)$ is $q^{2}\left(q^{3}+1\right) /(q+1)$, and therefore the involution centralizer has order $q(q-1)(q+1)$. Hence $2 \times L_{2}(q)$ is the full involution centralizer.

At this stage we have proved that our group $R(q)$ is simple (if $q>3$ ) of order $q^{3}\left(q^{3}+1\right)(q-1)$ and that it has an involution with centralizer $2 \times L_{2}(q)$. In the usual terminology of the literature on the Classification Theorem for Finite Simple Groups, therefore, we have 'constructed' (i.e. proved the existence of) the Ree simple groups of type ${ }^{2} G_{2}$.

To prove that our groups are isomorphic to the Ree groups as usually defined, however, is a different story. This so-called 'uniqueness' problem for the Ree groups was a major stumbling-block in the proof of the Classification Theorem for Finite Simple Groups, solved eventually by Bombieri [1]. An alternative strategy, which proves a good deal less, is to observe that our generators are the same as in [5], up to a suitable change of signs on the basis $\left\{v_{r}\right\}$, so by Lie theory our groups are the Ree groups.

\section{Connections with Lie theory}

When I gave a talk on this construction at the Scottish Algebra Day in St Andrews (28th April 2006), Meinolf Geck asked me whether (analogous to his description of the Suzuki groups in [4]) this method also provides an explicit description of the graph automorphism of $G_{2}(F)$, which makes it obvious that when $F$ is algebraically closed this is a homomorphism (but not an isomorphism!) of algebraic groups, and that it squares to the standard Frobenius. Indeed it does.

To see this, observe that, with respect to the basis $\left\{v_{r} \wedge v_{s}\right\}$, the matrix entries in the action of $G_{2}(F)$ on $\Lambda^{2}(V)$ are polynomials in the matrix entries in the action on $V$. (They are of course $2 \times 2$ determinants.) Now change basis on $\Lambda^{2}(V)$ to include the basis $\left\{v_{r}^{\prime}, v_{r}^{*}\right\}$ given above for $W$. Such a basis change can be achieved by an invertible matrix over $\mathbb{F}_{3}$, so the matrix entries with respect to this new basis are still polynomials. Finally, the matrix entries for the action on $V^{*}$ are just a subset of the matrix entries we have just created. The projection $\rho$ then identifies $V^{*}$ with $V$ and produces the required group homomorphism.

Now perform this operation explicitly on the diagonal matrices

$$
\operatorname{diag}\left(\lambda_{1}, \lambda_{2}, \lambda_{1} \lambda_{2}^{-1}, 1, \lambda_{1}^{-1} \lambda_{2}, \lambda_{2}^{-1}, \lambda_{1}^{-1}\right) .
$$

Using the basis $v_{-3}^{*}, \ldots, v_{3}^{*}$ of $V^{*}$ defined by Table 1 , we obtain the matrix

$$
\operatorname{diag}\left(\lambda_{1} \lambda_{2}, \lambda_{1}^{2} \lambda_{2}^{-1}, \lambda_{1}^{-1} \lambda_{2}^{2}, 1, \lambda_{1} \lambda_{2}^{-2}, \lambda_{1}^{-2} \lambda_{2}, \lambda_{1}^{-1} \lambda_{2}^{-1}\right) .
$$

and if we perform the same operation again we obtain

$$
\operatorname{diag}\left(\lambda_{1}^{3}, \lambda_{2}^{3}, \lambda_{1}^{3} \lambda_{2}^{-3}, 1, \lambda_{1}^{-3} \lambda_{2}^{3}, \lambda_{2}^{-3}, \lambda_{1}^{-3}\right) .
$$

Finally, the subgroup $2^{3}: 7: 3$ is represented by identical matrices, with entries in $\mathbb{F}_{3}$, on $V$ and $V^{*}$, and this subgroup together with the maximal torus generates $G_{2}(q)$. Thus the given automorphism squares to the Frobenius, as claimed.

Of course, the Lie theory is never far away from what we have been doing. Indeed, $W$ is essentially the Lie algebra, and $\left\{v_{r}^{\prime}, v_{r}^{*}\right\}$ is a Chevalley basis. But the point is that we do not need to use the Lie algebra structure. One way of looking at what we have done is that we have factored the comultiplication $\mu$ on the octonion algebra $V$ through the submodule $W$ of $\Lambda^{2}(V)$. As a $G_{2}(F)$-module, 
$\Lambda^{2}(V)$ is a uniserial module with composition factors $V, V^{*}, V$, so there is a 14dimensional quotient $W^{*}$ of $\Lambda^{2}(V)$, and a corresponding way to factor the product $m$ through $W^{*}$. (Our projection map $\rho$ should therefore 'really' be written as a map $W^{*} \rightarrow V$, but this would merely shift the difficulty onto constructing an $H$-module isomorphism between $W$ and $W^{*}$.) Putting together all the relevant $G_{2}(F)$-module homomorphisms we obtain a commutative diagram as follows:

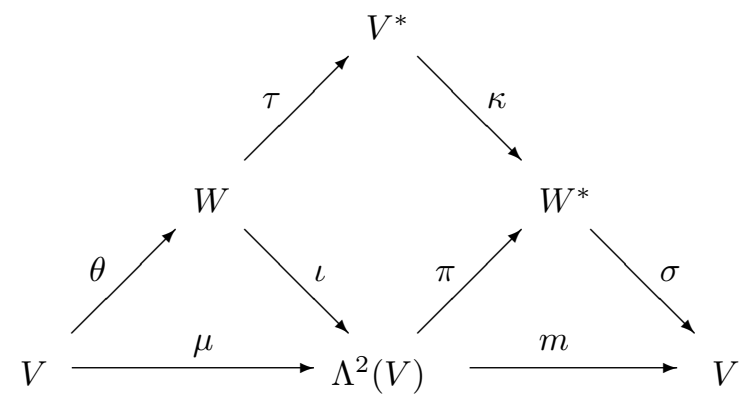

In view of the fact that this diagram contains a total of eight multiplications, comultiplications, and left/right semi-(co-)multiplications, I feel justified in renaming the octonion algebra in characteristic 3 as the octopus algebra. Actually, I have left out the most important part of the octopus algebra, namely the 'middle bi-semi-multiplication' $\mathfrak{m}:=\pi \iota=\kappa \tau$ which forms the heart of the exact sequence

$$
0 \longrightarrow V \stackrel{\theta}{\longrightarrow} W \stackrel{\mathfrak{m}}{\longrightarrow} W^{*} \stackrel{\sigma}{\longrightarrow} V \longrightarrow 0
$$

or perhaps the mouth of the octopus.

Changing our viewpoint slightly, we can shift the field automorphism into the identification of $W$ with $W^{*}$, so that (since $\theta$ is a natural inclusion and $\sigma$ is a natural projection) the structure which defines the Ree groups inside $G_{2}(F)$ is completely encapsulated in the resulting 'twisted middle bi-semi-multiplication' on $W$. In a sense, we have now come full circle. The twisted middle bi-semi-multiplication has the simple form $\mathfrak{m}^{*}: \sum \lambda_{r}^{*} v_{r}^{*}+\sum \mu_{r} v_{r}^{\prime} \mapsto \sum \lambda_{r} v_{r}^{\prime}$ with respect to the Chevalley basis of the Lie algebra $W$, and ${ }^{2} G_{2}(F)$ may be defined as the group of automorphisms of the Lie algebra which commute with $\mathfrak{m}^{*}$.

\section{References}

[1] E. Bombieri, A. Odlyzko, D. Hunt, Thompson's problem $\left(\sigma^{2}=3\right)$. Appendices by A. Odlyzko and D. Hunt. Invent. Math. 58 (1980), 77-100.

[2] J. J. Cannon et al. The Magma programming language, Version 2.11. School of Mathematics and Statistics, University of Sydney (2004).

[3] R. W. Carter, Simple groups of Lie type, Wiley, 1972.

[4] M. Geck, Introduction to algebraic groups, Oxford University Press, 2004.

[5] R. B. Howlett, L. J. Rylands and D. E. Taylor, Matrix generators for exceptional groups of Lie type. J. Symbolic Comput. 31 (2001), 429-445.

[6] R. Ree, A family of simple groups associated with the simple Lie algebra of type $G_{2}$, Amer. J. Math. 83 (1961), 432-462.

[7] L. J. Rylands and D. E. Taylor, Constructions for octonion and exceptional Jordan algebras, Designs, codes and cryptography 21, 191-203. 
[8] J. Tits, Les groupes simples de Suzuki et Ree, Séminaire Bourbaki 1960/61, exp. 210.

[9] H. van Maldeghem, Generalized polygons. Monographs in Mathematics, 93. Birkhäuser, Basel, 1998.

[10] R. A. Wilson, An elementary construction of the large Ree groups ${ }^{2} F_{4}(q)$, in preparation.

\section{Appendix}

Here we give the details of the calculations. First we show that the basis of $W$ given in Table 1 has the required properties. To save space we use a shorthand notation, so that $s t$ denotes $i_{s} \wedge i_{t}$. We use the fact that $-s t=t s$ to remove minus signs, and use a dot to denote addition. First we calculate $v_{r}^{\prime}=\theta\left(v_{r}\right)$.

$$
\begin{aligned}
& v_{-3}^{\prime}=-i_{3}^{\prime}-i_{5}^{\prime}-i_{6}^{\prime}=64.25 .10 .16 .40 .32 .20 .51 .43 \\
& v_{0} \wedge v_{-3}+v_{-2} \wedge v_{-1}=31.51 .61 .23 .25 .26 .10 .13 .61 .20 .23 .62 .40 .43 .64 \\
& =51.16 .32 .25 \cdot 10.20 .40 .43 .64 \\
& v_{-2}^{\prime}=-i_{1}^{\prime}-i_{2}^{\prime}-i_{4}^{\prime}=42.03 \cdot 65.53 .14 .06 .05 .36 .21 \\
& v_{1} \wedge v_{-3}+v_{-2} \wedge v_{0}=03.05 .06 .53 .63 .63 .65 .12 .14 .12 .42 \\
& =03.05 .06 .53 .36 .65 .21 .14 .42 \\
& v_{-1}^{\prime}=-i_{0}^{\prime}-i_{3}^{\prime}+i_{6}^{\prime}=31.62 .54 .64 \cdot 25 \cdot 10.02 .15 .34 \\
& v_{-3} \wedge v_{2}+v_{-1} \wedge v_{0}=13.15 .16 .23 .25 .26 .34 .54 .64 .10 .13 .61 .02 .32 .26 \\
& =31.16 .25 .62 .34 .54 .64 .10 .02 \\
& v_{0}^{\prime}=i_{1}^{\prime}-i_{2}^{\prime}=24.30 .56 .53 .14 .06 \\
& v_{-3} \wedge v_{3}+v_{2} \wedge v_{-2}+v_{1} \wedge v_{-1}=35.36 .35 .65 .63 .65 .21 .41 .12 .42 .41 .42 .03 .60 .03 .36 .60 .63 \\
& =53.56 .14 .24 .30 .06 \\
& v_{1}^{\prime}=-i_{0}^{\prime}+i_{3}^{\prime}-i_{6}^{\prime}=31.62 .54 .46 .52 .01 .20 .51 .43 \\
& v_{3} \wedge v_{-2}+v_{0} \wedge v_{1}=13.23 .43 .51 .52 .54 .16 .26 .46 .01 .13 .61 .20 .32 .26 \\
& \begin{aligned}
& =13 \cdot 43 \cdot 51 \cdot 52.54 \cdot 62 \cdot 46 \cdot 01.20 \\
v_{2}^{\prime}=i_{1}^{\prime}+i_{2}^{\prime}-i_{4}^{\prime} & =24.30 \cdot 56 \cdot 35 \cdot 41 \cdot 60 \cdot 05 \cdot 36.21
\end{aligned} \\
& v_{-1} \wedge v_{3}+v_{0} \wedge v_{2}=30.05 .60 .35 .63 .63 .56 .12 .41 .12 .24 \\
& =30.05 .60 .35 .36 .56 \cdot 21.41 .24 \\
& v_{3}^{\prime}=i_{3}^{\prime}-i_{5}^{\prime}+i_{6}^{\prime}=46.52 .01 \cdot 16.40 .32 .02 .15 .34 \\
& v_{3} \wedge v_{0}+v_{2} \wedge v_{1}=31.15 .61 .23 .52 .26 .01 .13 .61 .02 .23 .62 .40 .34 .46 \\
& =15.16 .32 .52 .01 .02 .40 .34 .46
\end{aligned}
$$

Next we verify that $\rho\left(v_{r}^{*}\right)=v_{r}$. Notice that the base change between the $i_{t}^{*}$ and the $v_{r}^{*}$ is only specified modulo $V^{\prime}$. Thus we have not specified the complete basis change on $W$. However, this can be obtained by analysing the calculations below in more detail. Expressions in brackets are vectors in $V^{\prime}$ which therefore cancel out.

$$
\begin{aligned}
v_{-3}^{*} \equiv-i_{3}^{*}-i_{5}^{*}-i_{6}^{*} & \equiv \text { } 64.52 .16 .04 .20 .15 \\
v_{-3} \wedge v_{-2} & =31.32 .34 .51 .52 .54 .61 .62 .64 \\
& =(31.62 .54) .34 .51 .32 .61 .52 .64 \\
& \equiv 34.51 \cdot(20.51 .43) .32 .61 .(61.04 .23) .52 .64 \\
& =15.20 .16 .04 .52 .64 \\
v_{-2}^{*} \equiv-i_{1}^{*}-i_{2}^{*}-i_{4}^{*} & =42.30 .53 .41 .05 .63 \\
v_{-1} \wedge v_{-3} & =03.05 .06 .35 .36 .36 .56 \\
& \equiv 03.56 \cdot(42.03 .65) .06 .35 .(35.41 .60) .05 .63 \\
v_{-1}^{*} \equiv-i_{0}^{*}-i_{3}^{*}+i_{6}^{*} & =30.42 .53 .41 .05 .63 \\
&
\end{aligned}
$$




$$
\begin{aligned}
& v_{-2} \wedge v_{1}=10.20 .40 .31 .32 .34 .16 .26 .46 \\
& \equiv \text { 31.26.10.46.(46.52.01).20.34.(20.51.43).(40.32.16) } \\
& \equiv 31.26 .64 .52 .02 .51 \\
& v_{0}^{*} \equiv i_{1}^{*}-i_{2}^{*}=24.03 .53 .41 \\
& v_{-3} \wedge v_{3}+v_{-2} \wedge v_{2}=35.36 .35 .65 .63 .65 .12 .14 .21 .24 .14 .24 \\
& \equiv \text { 53.56.41.42.(42.03.65) } \\
& =53.41 .24 .03 \\
& v_{1}^{*} \equiv-i_{0}^{*}+i_{3}^{*}-i_{6}^{*}=31.26 \cdot 46.25 .20 .15 \\
& v_{2} \wedge v_{-1}=01.31 .16 .02 .32 .26 .40 .43 .64 \\
& \equiv \text { 31.26.(16.40.32).01.64.(64.25.10).02.43.(02.15.34) } \\
& =31.26 .46 .25 .20 .15 \\
& v_{2}^{*} \equiv i_{1}^{*}+i_{2}^{*}-i_{4}^{*}=24.03 .35 .14 .05 .63 \\
& v_{1} \wedge v_{3}=30.05 .60 .53 .36 .36 .65 \\
& \equiv \text { 30.65.(24.30.56).60.53.(53.14.06).05.63 } \\
& =03.24 .35 .14 .05 .63 \\
& v_{3}^{*} \equiv i_{3}^{*}-i_{5}^{*}+i_{6}^{*}=46.25 .16 .04 .02 .51 \\
& v_{3} \wedge v_{2}=31.32 .43 .15 .25 .54 .61 .62 .46 \\
& \equiv(31.62 .54) \cdot 25.46 .32 .61 .(61.04 .23) .43 .15 .(02.15 .34) \\
& \equiv 25.46 .16 .04 .51 .02
\end{aligned}
$$

Next we verify that $s_{3}$ is in the Ree group. Here we write $r$ for $v_{r}$ if $r$ is nonnegative, and $\bar{r}$ for $v_{-r}$ if $r$ is positive. We use the same trick to eliminate signs as above. First we verify that the action on $v_{r}^{\prime}$ is correct.

$$
\begin{aligned}
& v_{-3}^{\prime}=0 \overline{3} \cdot \overline{2} \overline{1} \mapsto 0 \overline{3} \cdot \overline{3} \overline{1} \cdot \overline{2} \overline{1} \cdot \overline{3} \overline{2} \cdot \overline{1} \overline{3} . \overline{2} \overline{3} \\
& =0 \overline{3} . \overline{2} \overline{1} \\
& v_{-2}^{\prime}=1 \overline{3} \cdot \overline{2} 0 \quad \mapsto \quad 1 \overline{3} \cdot 0 \overline{3} \cdot \overline{1} \overline{3} \cdot \overline{2} 0 \cdot \overline{1} \overline{2} \cdot 0 \overline{3} \cdot \overline{3} \overline{1} \\
& =1 \overline{3} \cdot \overline{2} 0 . \overline{3} 0 . \overline{1} \overline{2} \\
& v_{-1}^{\prime}=\overline{3} 2 . \overline{1} 0 \mapsto \overline{3} 2 \cdot 1 \overline{3} \cdot 0 \overline{3} \cdot \overline{1} \overline{3} \cdot \overline{1} 0 \cdot \overline{2} 0 \cdot 0 \overline{3} \cdot \overline{1} \overline{2} \cdot \overline{3} \overline{1} \\
& =\overline{3} 2 \cdot \overline{1} 0.1 \overline{3} \cdot \overline{2} 0 \cdot \overline{3} 0 \cdot \overline{1} \overline{2} \\
& v_{0}^{\prime}=\overline{3} 3.2 \overline{2} .1 \overline{1} \quad \mapsto \quad \overline{3} 3 . \overline{3} 2 . \overline{1} \overline{3} . \overline{3} \overline{2} .2 \overline{2} . \overline{2} 1 . \overline{2} 0 . \overline{2} \overline{1} . \overline{3} \overline{2} . \overline{3} 2.1 \overline{3} .0 \overline{3} . \overline{1} \overline{3} .1 \overline{1} .1 \overline{2} . \overline{3} 1.0 \overline{1} .0 \overline{2} \cdot \overline{3} 0 . \overline{1} \overline{2} . \overline{3} \overline{1} .3 \overline{1} . \overline{3} \overline{2} \\
& =\overline{3} 3.2 \overline{2} .1 \overline{1} .2 \overline{3} .0 \overline{1} \\
& v_{1}^{\prime}=3 \overline{2} .01 \quad \mapsto \quad 3 \overline{2} .2 \overline{2} \cdot \overline{2} \overline{1} \cdot \overline{2} \overline{3} \cdot \overline{3} 3 . \overline{3} 2 . \overline{1} \overline{3} \cdot \overline{3} \overline{2} \cdot 01.0 \overline{1} \cdot 0 \overline{3} \cdot 1 \overline{1} \cdot 0 \overline{1} . \overline{3} \overline{1} \\
& =3 \overline{2} .01 . \overline{3} 3.2 \overline{2} \cdot 1 \overline{1} \cdot \overline{3} 2 . \overline{1} 0.0 \overline{3} \cdot \overline{2} \overline{1} \\
& v_{2}^{\prime}=\overline{1} 3.02 \mapsto \overline{1} 3 . \overline{1} 2 . \overline{1} \overline{2} \cdot \overline{3} \overline{1} . \overline{2} 3 . \overline{2} 2 . \overline{2} \overline{2} . \overline{3} \overline{2} .3 \overline{3} \cdot 2 \overline{3} \cdot \overline{3} \overline{1} . \overline{2} \overline{3} \cdot 02.10 . \overline{1} 0.0 \overline{3} .2 \overline{1} \cdot \overline{1} 1 . \overline{1} 0 . \overline{3} \overline{1} \\
& =\overline{1} 3.02 . \overline{2} 3.10 .3 \overline{3} \cdot \overline{2} 2 . \overline{1} 1.2 \overline{3} \cdot 0 \overline{1} \cdot 0 \overline{3} \cdot \overline{2} \overline{1} \\
& v_{3}^{\prime}=30.21 \mapsto \quad 30.20 .0 \overline{1} \cdot \overline{2} 0.0 \overline{3} \cdot \overline{1} 3 . \overline{1} 2 . \overline{1} \overline{2} . \overline{3} \overline{1} .21 .10 .1 \overline{1} . \overline{3} 1.20 .01 .0 \overline{1} \cdot \overline{3} 0.2 \overline{1} . \overline{1} 1 . \overline{1} 0 . \overline{3} \overline{1} .2 \overline{3} . \overline{3} 1 . \overline{3} 0 . \overline{3} \overline{1} \\
& =\quad 30.21 \cdot \overline{1} 3 \cdot 02 \cdot 2 \overline{3} \cdot 0 \overline{1} \cdot 1 \overline{3} \cdot \overline{2} 0 . \overline{3} 0 . \overline{1} \overline{2}
\end{aligned}
$$

Finally we verify the action of $s_{3}$ on the $v_{r}^{*}$, modulo $V^{\prime}$. As before, expressions in brackets are vectors in $V^{\prime}$.

$$
\begin{aligned}
& v_{-3}^{*}=\overline{3} \overline{2} \mapsto \overline{3} \overline{2} \\
& v_{-2}^{*}=\overline{1} \overline{3} \mapsto \overline{1} \overline{3} . \overline{2} \overline{3} \\
& v_{-1}^{*}=\overline{2} 1 \mapsto \overline{2} 1 . \overline{2} 0 . \overline{2} \overline{1} \cdot \overline{2} \overline{3} \cdot 1 \overline{3} \cdot 0 \overline{3} \cdot \overline{1} \overline{3} \\
& =\overline{2} 1 \cdot \overline{1} \overline{3} \cdot \overline{2} \overline{3} \cdot(1 \overline{3} \cdot \overline{2} 0) \cdot(0 \overline{3} \cdot \overline{2} \overline{1}) \\
& v_{0}^{*}=\overline{3} 3 . \overline{2} 2 \mapsto \overline{3} 3 . \overline{3} 2 . \overline{1} \overline{3} \cdot \overline{3} \overline{2} \cdot \overline{2} 2.1 \overline{2} \cdot 0 \overline{2} \cdot \overline{1} \overline{2} \cdot \overline{2} \overline{3} \cdot 2 \overline{3} \cdot \overline{3} 1 . \overline{3} 0 . \overline{3} \overline{1} \\
& =\overline{3} 3 \cdot \overline{2} 2 \cdot 1 \overline{2} \cdot(\overline{3} 0 . \overline{1} \overline{2}) \cdot(\overline{3} 1.0 \overline{2}) \\
& v_{1}^{*}=2 \overline{1} \quad \longmapsto \quad 2 \overline{1} \cdot \overline{1} 1 . \overline{1} 0 . \overline{3} \overline{1} .2 \overline{2} \cdot \overline{2} 1 . \overline{2} 0 . \overline{2} \overline{1} \cdot \overline{3} \overline{2} \cdot \overline{3} 2.1 \overline{3} \cdot 0 \overline{3} \cdot \overline{1} \overline{3} \\
& =\quad 2 \overline{1} \cdot \overline{3} 3 \cdot \overline{2} 2 \cdot \overline{2} 1 \cdot \overline{3} \overline{2} \cdot(3 \overline{3} \cdot \overline{2} 2 \cdot \overline{1} 1) \cdot(\overline{3} 2 \cdot \overline{1} 0) \cdot(1 \overline{3} \cdot \overline{2} 0) \cdot(0 \overline{3} \cdot \overline{2} \overline{1}) \\
& v_{2}^{*}=13 \mapsto \quad 13.12 \cdot \overline{1} 1.1 \overline{1} \cdot \overline{3} 1.03 \cdot 02 \cdot \overline{1} 0.0 \overline{2} \cdot \overline{3} 0 . \overline{1} 3 . \overline{1} 2 \cdot \overline{1} \overline{2} \cdot \overline{3} \overline{1} \cdot \overline{3} 3 . \overline{3} 2 . \overline{1} \overline{3} \cdot \overline{3} \overline{2} \\
& =13 \cdot \overline{1} 2.3 \overline{3} \cdot 2 \overline{2} \cdot 1 \overline{2} \cdot \overline{3} \overline{2} \cdot(03.12) \cdot(\overline{1} 3 \cdot 02) \cdot(3 \overline{3} 3 \cdot \overline{2} 2 \cdot \overline{1} 1) \cdot(\overline{3} 2 \cdot \overline{1} 0) \cdot(\overline{3} 1.0 \overline{2}) \cdot(\overline{3} 0 . \overline{1} \overline{2}) \\
& v_{3}^{*}=32 \quad \mapsto \quad 32.2 \overline{1} . \overline{2} 2.2 \overline{3} .13 .12 . \overline{1} 1.1 \overline{2} . \overline{3} 1.03 .02 . \overline{1} 0.0 \overline{2} . \overline{3} 0 . \overline{1} 3 \cdot \overline{1} 2 . \overline{1} \overline{2} . \overline{3} \overline{1} .3 \overline{3} .2 \overline{3} \cdot \overline{3} \overline{1} . \overline{2} \overline{3} \\
& =32.13 \cdot 1 \overline{2} \cdot \overline{1} \overline{3} \cdot \overline{2} \overline{3} \cdot(3 \overline{3} \cdot \overline{2} 2 \cdot \overline{1} 1) \cdot(03 \cdot 12) \cdot(\overline{1} 3.02) \cdot(3 \overline{2} \cdot 01) \cdot(\overline{3} 1.0 \overline{2}) \cdot(\overline{3} 0 . \overline{1} \overline{2})
\end{aligned}
$$

